\title{
Quantitative Modelling of Electron Spectroscopy Intensities for Supported Nanoparticles: The Hemispherical Cap Model for Non-Normal Detection
}

\author{
James C. Sharp* and Charles T. Campbell* \\ Department of Chemistry \\ University of Washington \\ Seattle, Washington 98195-1700, USA
}

\begin{abstract}
Nanoparticles of one element or compound dispersed across the surface of another substrate element or compound form the basis for many materials of great technological importance, such as heterogeneous catalysts, fuel cells and other electrocatalysts, photocatalysts, chemical sensors and biomaterials. They also form during film growth by deposition in many fabrication processes. The average size and number density of such nanoparticles is often very important, and these can be estimated with electron microscopy or scanning tunneling microscopy. However, this is very time consuming and often unavailable with sufficient resolution when the particle size is $\sim 1 \mathrm{~nm}$. Because the probe depth of electron spectroscopies like X-Ray Photoelectron Spectroscopy (XPS) or Auger Electron Spectroscopy (AES) is $\sim 1 \mathrm{~nm}$, these provide quantitative information on both the total amount of adsorbed material when it is in the form of such small nanoparticles, and the particle thickness. For electron spectroscopy conducted with electron detection normal to the surface, Diebold et al. ${ }^{1}$ derived analytical relationships between the signal intensities for the adsorbate and substrate and the particles' average size and number density, under the assumption that all the particles have hemispherical shape and the same radius. In this paper, we report a simple angle- and particle-size-dependent correction factor that can be applied to these analytical expressions so that they can also be extended to measurements made at other detection angles away from the surface normal. This correction factor is computed using numerical integration and presented for use in future modeling. This correction factor is large $(>2)$ for angles beyond $60^{\circ}$, so comparing model predictions to measurements at both $0^{\circ}$ and $\geq 60^{\circ}$ will also provide a new means for testing the model's assumptions (hemispherical shape and fixed size particles). The ability to compare the hemispherical cap model at several angles simultaneously also should enable more accurate estimates of surface structural parameters when elastic diffraction effects cause strong peaks in the angular distributions of emitted electrons.
\end{abstract}

* Corresponding authors: emails=sharp275@uw.edu and charliec@uw.edu 
A very common structural motif in materials science involves nanoparticles of one material dispersed across the surface of another. Such structures are commonly encountered when making and/or studying catalysts, microelectronics, plasmonic and optical devices, nanoelectrode arrays, sensors, coatings and thin film growth by vapor deposition or other condensation processes. For example, metal nanoparticles dispersed across the surfaces of oxides and carbon supports are key ingredients in many heterogeneous catalysts, fuel cells, other electrocatalysts and photocatalysts, all of which are crucial for energy, fuel and environmental technologies and chemical processing. One of the three growth modes that occur during thin film growth by vapor deposition is the Volmer-Weber mode, whereby clusters of the deposited material first nucleate and then grow as 3-D nanoparticles on the substrate. ${ }^{1-8}$ A very common method to estimate the size and number density of such nanoparticles is based upon measurements of the intensities of peaks associated with substrate and adsorbate elements using an electron spectroscopy, typically either XPS or AES. A model that has been very widely applied for analyzing these intensities is the hemispherical cap model introduced by Diebold et al., ${ }^{1}$ which assumes that the nanoparticles all have the shape of hemispheres with the same diameter. It is often applied to cases where the intensities of substrate and adsorbate peaks have been measured versus the amount of adsorbate deposited on the flat substrate surface, in which case the further assumption is made that the number of particles per unit area remains constant. This number density is estimated from a fit to these plots of intensities versus coverage (or the intensities at any one coverage) to the resulting equations of this hemispherical cap model, which also gives the average diameter of the particles at each coverage. ${ }^{1,6}$ Unfortunately, these equations for the hemispherical cap model only apply when the electron intensities have been measured for a take-off angle normal to the substrate surface. Here, we present an extension of that model that allows it to be applied at other angles of electron detection, which are often demanded by the constraints on sample mounting or manipulation imposed by the particular vacuum chamber being used. This ability to model measurements at several different angles should also mitigate the complications associated with strong peaks in the angular distributions of electrons due to diffraction effects.

In either XPS or AES, electrons are emitted from a material due to interaction with either $\mathrm{X}$-Ray photons or high energy electrons. The intensity of the electrons emitted at given kinetic energies is element specific. These electrons can interact with matter prior to escaping vacuum 
and lose energy. The probability for an electron to traverse through matter without significant

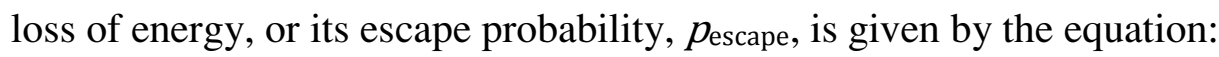

$$
p_{\text {escape }}=e^{-d / \lambda}
$$

where $d$ is the distance the electron transverses through matter and $\lambda$ is the characteristic attenuation length for the measured electron. The value of $\lambda$ depends on its kinetic energy and the material through which it is passing. It is usually approximated as equal to the inelasticmean-free-path (IMFP), which has been tabulated in detail as a function of kinetic energy and material. ${ }^{9}$ However, that approximation neglects elastic scattering effects like those that give rise to forward-focusing peaks is photoelectron diffraction. Therefore, it has been shown that a better approximation is to use the so-called "effective-attenuation-length" (EAL) for $\lambda$ instead, ${ }^{10,11}$ which has also been tabulated as a function of kinetic energy, material and other parameters. ${ }^{12}$ Those tables show that the EAL can differ by up to $35 \%$ from the IMFP for common XPS and AES measurement conditions, but they are usually closer.

Using Equation 1 for a simple flat overlayer of an adsorbate of uniform thickness, $t$, on a substrate, the ratio of the intensity of electrons from an element of the substrate, $I_{\text {sub }}$, to its intensity with no overlayer, $I_{s u b, 0}$, for normal detection is given by ${ }^{13}$ :

$$
I_{\text {sub,normal }} / I_{\text {sub,normal }, 0}=e^{-t / \lambda_{\text {sub }}} \text {, }
$$

where $\lambda_{s u b}$ is the IMFP or EAL of electrons from the substrate element's peak through the adsorbate material. Similarly, the ratio of the intensity of electrons from an overlayer, Iads, to the intensity of electron from an infinitely thick adsorbate overlayer, $I_{a d s}, \infty$, at normal detection is ${ }^{13}$ :

$$
I_{\text {ads }, \text { normal }} / I_{\text {ads,normal }, \infty}=1-e^{-t / \lambda_{a d s}},
$$

where $\lambda_{a d s}$ is the IMFP or EAL of electrons from the adsorbate element's peak through the adsorbate material. When the angle of detection is instead at some angle $\theta$ from normal to the surface, the thickness, $t$, is replaced with $t / \cos \theta$ in these equations ${ }^{13}$. 
When the adsorbate is in the form of uniform-size hemispherical nanoparticles instead of a uniformly thick layer, the analysis is much more complex, since the distance any electron travels through the solids is dependent upon where the electron originated from within the plane of the substrate surface (e.g., how thick the particle is at that location). In the hemispherical cap model mentioned above, the ratio of the intensity of emitted electrons from adsorbed hemispherical particles to the intensity of emitted electrons from an infinitely thick film of the same adsorbate for the case of normal detection was calculated by Diebold et al. by averaging (integrating) over the total surface area, and found to be ${ }^{1}$ :

$$
\frac{I_{a d s, n o r m a l}}{I_{a d s, n o r m a l, \infty}}=n \pi R^{2}-2 \pi n \lambda_{a d s}^{2}\left[1-\left[1+\frac{R}{\lambda_{a d s}}\right] e^{-R / \lambda_{a d s}}\right]
$$

where $n$ is the particle density, and $R$ is the radius of the hemispherical caps. For the signal from the substrate relative to its signal at zero adsorbate coverage, they found that ${ }^{1}$ :

$$
\frac{I_{\text {sub }, \text { normal }}}{I_{\text {sub }, \text { normal }, 0}}=1-n \pi R^{2}-2 \pi n \lambda_{\text {sub }}^{2}\left[1-\left[1+\frac{R}{\lambda_{\text {sub }}}\right] e^{-R / \lambda_{\text {sub }}}\right]
$$

These equations are only valid for electrons detected normal to the surface.

In order to determine the relationship between particle geometry and signal intensity of electron spectroscopies using non-normal detection methods, equations similar to Equations 4 and 5 can be derived for other angles by properly integrating the escape probability given in Equation 1. Consider a hemispherical adsorbate particle centered at $(0,0,0)$, where its interface with the support substrate surface is defined as the $\mathrm{z}=0$ plane, as shown in Fig. 1. For detection at some angle $\theta$ from the surface normal as shown, the distance an electron travels through the adsorbate from a single coordinate is no longer simply related to the $\mathrm{z}$-coordinate, or the $\mathrm{z}$ coordinate divided by $\cos \theta$. Figure 1 illustrates, for example, how a simple $R / \cos \theta$ correction would misrepresent the distance the electrons travel through the adsorbate material. Next we describe how one can solve for signal intensities using a simple numerical integration over the surface and sample volume.

The substrate surface is divided up into tiny increments (i.e., differential area elements dxdy), each located at some $x, y$ coordinate in the $\mathrm{z}=0$ plane. For a single area increment on the substrate surface located at point $\left(\mathrm{x}_{0}, \mathrm{y}_{0}, 0\right)$, the distance that an electron emitted from that point 
would travel through the hemispherical cap in the direction of the detector is calculated. For any point on the surface directly underneath the hemispherical cap, this distance is simply the length of the line originating from that point $\left(\mathrm{x}_{0}, \mathrm{y}_{0}, 0\right)$, aiming toward the detector, and stopping at the point where it intersects the top surface of the hemispherical cap, which is defined by the equation for the sphere: $\mathrm{x}^{2}+\mathrm{y}^{2}+\mathrm{z}^{2}=R^{2}$. For each such starting point on the substrate surface, the $(\mathrm{x}, \mathrm{y}, \mathrm{z})$ values of this point on the hemisphere's surface is calculated for the chosen values of $R / \lambda$, and $\theta$. (To generalize, this was actually done for a given value of $R / \lambda$, treating $\mathrm{x}, \mathrm{y}$ and $\mathrm{z}$ in "reduced length" units, $\mathrm{x} / \lambda, \mathrm{y} / \lambda$ and $\mathrm{z} / \lambda$.) Since $\mathrm{y}=\mathrm{y}_{0}$, this simply requires solving for the only two unknowns ( $\mathrm{x}$ and $\mathrm{z}$ ) using two equations: the equation for the sphere and the equation for the line that goes from $\left(\mathrm{x}_{0}, \mathrm{y}_{0}, 0\right)$ to the detector. The distance the electron travels is then simply the distance between these two points $\left(\mathrm{x}_{0}, \mathrm{y}_{0}, 0\right)$ and $\left(\mathrm{x}_{1} \mathrm{y}_{0}, \mathrm{z}\right), d=\sqrt{\left(x-x_{0}\right)^{2}+(z-0)^{2}}$. For points on the substrate in the "shadow" of the particle (as viewed from the detector, see Fig. 1), the line originating from that point to the detector intercepts the top surface of the hemisphere twice, so there are two solutions to these equations. The distance between these two intersection points (1 and 2) is just $d=\sqrt{\left(x_{1}-x_{2}\right)^{2}+\left(z_{1}-z_{2}\right)^{2}}$, and it equals the distance an electron from the substrate surface travels through the hemispherical cap. These distances are used with Eq. (1) to calculate the escape probability of substrate electrons, $p_{\text {escape, }}$ from every area increment on its surface. There is no attenuation length of substrate electrons $\left(d=0\right.$ and $\left.p_{\text {escape }}=1\right)$ when they originate from other areas of the substrate surface not blocked by the particle. The area-average value of $p_{\text {escape }}$ is calculated by numerically integrating it over the substrate surface and dividing by the area. This average requires using the number of such particles per unit area, $n$, and assumes that this number density is so low that the covered and shadowed areas from two different particles have insignificant probability for overlap. Assuming the particles are distributed on the surface hexagonally, the maximum radius they can reach before the spectroscopic footprints from two particles overlap is:

$$
R_{\max }=2 \sqrt{1 /(2 n \sqrt{3})} /(1+1 / \cos \theta)
$$

Once the area-average escape probability is calculated in this way, the substrate signal is just given by its value in the absence of any adsorbate particles multiplied by this average $p_{\text {escape }}$. Note that $\lambda_{\text {sub }}$ is used for the above calculations. 
These same distances and numerical integration method are used to calculate the signal from the adsorbate, only now the escape probability in Eq. (1) must be calculated using $\lambda_{a d s}$ instead, and now it also must be integrated over this distance $d$ and divided by that same integral over infinite distance. This gives the ratio of the adsorbate signal from any given volume element in the hemisphere (defined by the differential area element $\mathrm{d} x \mathrm{~d} y$ at $(\mathrm{x}, \mathrm{y})$ on the substrate surface translated up through the particle at angle $\theta$ from normal in the direction toward the detector through a total distance $d$ of adsorbate material. This ratio is then averaged over the whole substrate surface area, to get the area-averaged signal from the adsorbate relative to the signal from an infinitely thick plate of pure adsorbate.

To verify that this methodology and the computer program to implement it were correct, the values calculated using this method at normal detection $(\theta=0)$ were compared to those calculated using Equation 4, as shown in Fig. 2. Using a step size $0.01 \mathrm{~nm}$ (i.e., the differential area element $\mathrm{d} x \mathrm{~d} y$ has an area of $0.0001 \mathrm{~nm}^{2}$ ) to calculate the signals from a collection of hemispherical caps relative to the signal calculated using instead Equation 4 leads to an error of less than $0.1 \%$. As usual with numerical integration, choosing a smaller step size leads to a greater accuracy while increasing the computational time. Changing the step size from $0.02 \mathrm{~nm}$ to $0.01 \mathrm{~nm}$ leads to less than $0.01 \%$ change in the calculated values.

Figure 3 shows a plot of the resulting correction factor, $f_{\theta, R / \lambda}=\left(I_{a d s, \theta} / I_{a d s, \theta, \infty}\right) /\left(I_{a d s, n o r m a l}\right.$ ( $I_{a d s, n o r m a l, \infty}$ ), that must be applied to Eq.(4) to calculate adsorbate signals measured at other detection angles $\theta$ (relative to the signal for an infinitely thick and continuous adsorbate film). This correction factor is independent of particle density $n$, provided the radius does not exceed $R_{\max }$ defined in Eq. (6), which does depend on $n$. Figure 3 shows that this correction factor for any given angle approaches a constant value for $R / \lambda>4$. This correction factor indeed asymptotically approaches the ratio of the projected area of the nanoparticle on the substrate surface as viewed from the detector (or spectroscopic footprint), which is given by the simple geometric relation:

$$
I_{\text {ads }, \theta} / I_{\text {ads,normal }}=0.5\left(1+\frac{1}{\cos \theta}\right) \text { for } R / \lambda>6 \text {. }
$$


The correction factor in Fig. 3 can be used as a simple scaling factor to correct the predictions of Eq. (4) for normal detection to any other angle of detection for measurements that are physically limited to detection angles other than normal. That is:

$$
I_{a d s, \theta} / I_{a d s, \theta, \infty}=\left(\frac{I_{a d s, \theta} / I_{a d s, \theta, \infty}}{I_{a d s, \text { normal }} / I_{\text {ads }, \text { normal }, \infty}}\right)\left(\frac{I_{a d s, \text { normal }}}{I_{\text {ads }, \text { normal }, \infty}}\right)=f_{\theta, R / \lambda}\left(\frac{I_{\text {ads }, \text { normal }}}{I_{\text {ads }, \text { normal }, \infty}}\right)
$$

Thus, one could, for example, use this to fit intensity versus adsorbate coverage data acquired at non-normal detection to estimate the particle density $n$, just as Eq. (4) has so frequently been used for normal detection. One must remember here that the $\lambda$ value to use in the $\mathrm{x}$-axis in Fig. 3 is the value appropriate for electrons at the kinetic energy of the adsorbate peak passing through the adsorbate material, or $\lambda_{a d s}$.

When measurements can be made at different detection angles, Fig. 3 could also be used together with measurements at different angles to test the validity of the assumptions of hemispherical shape and fixed size. Since this correction factor is fairly large $(>2)$ for angles above $60^{\circ}$, such a validity test would be most rigorous if applied to measurements at both normal detection and $>60^{\circ}$. If the model assumptions are correct, both angles should yield the same parameters (e.g., $n$ and/or $R$ ). Such a test would only be very sensitive to the average height : width ratio of the particles, and not their exact hemispherical shape.

Figure 3 can also be used together with Eq. (5) to model the signal from the substrate at any given detection angle. For this, the ratio of the substrate signal to that from the same substrate with no adsorbate, given by Eq. (5) for normal detection, is now instead given for any detection angle by:

$$
\frac{I_{s u b, \theta}}{I_{s u b, \theta, 0}}=1-f_{\theta, R / \lambda}\left(1-\frac{I_{\text {sub,normal }}}{I_{\text {sub }, \text { normal }, 0}}\right)
$$

where $f_{\theta, R / \lambda}$ is still simply read from Figure 3, but now using the $\lambda$ value for the bottom axis appropriate for electrons at the kinetic energy of the substrate peak passing through the adsorbate material (i.e., $\left.\lambda_{s u b}\right)$, instead of electrons at the adsorbate peak's kinetic energy $\left(\lambda_{a d s}\right)$. The ratio $I_{\text {sub,normal }} / I_{\text {sub,normal, }}$ here is obtained from Eq. (5). 
In conclusion, we have applied numerical integration to determine the normalized electron spectroscopy intensities for both the adsorbate and the substrate signals for an array of adsorbed hemispherical caps. We verified this method by comparing the numerical method with the analytical result derived previously for only normal detection ${ }^{1}$ and found less than $0.1 \%$ error. (This tiny error reflects the accuracy of the mathematical integrations, but not the accuracy of the physical assumption inherent in Eq. (1), which could easily lead to errors of $\sim 15 \%$ in particle size.) Thus, Fig. 3 combined with Eqs. (4), (5), (8) and (9) provide a simple and accurate method to model experimental signals in electron spectroscopies measured at nonnormal detection angles with the hemispherical cap model for adsorbates on substrates, and relate these signals to the size and number density of nanoparticles on the surface. The correction factors in Fig. 3 are well approximated with the function

$$
f_{\theta, R / \lambda}=A \exp \left(-(R / \lambda) / R^{\prime}\right)+B
$$

where $A$ and $R^{\prime}$ are fit parameters and $B$ is the asymptotic value given by Eq. (7). The data in Table 1 lists values for $A, R^{\prime}$, and $B$ that will fit the curves in Fig 3 with a maximum error of $>3 \%$.

\begin{tabular}{|c|c|c|c|}
\hline Angle from normal & $\boldsymbol{A}$ & $\boldsymbol{R}^{\prime}$ & $\boldsymbol{B}$ \\
\hline $0^{\circ}$ & $\boldsymbol{0}$ & - & $\mathbf{1}$ \\
\hline $30^{\mathrm{o}}$ & 0.0903 & .757 & 1.08 \\
\hline $45^{\mathrm{o}}$ & .241 & .876 & 1.21 \\
\hline $60^{\circ}$ & .545 & 1.08 & 1.50 \\
\hline $70^{\mathrm{o}}$ & 1.01 & 1.23 & 1.96 \\
\hline $75^{\mathrm{o}}$ & 1.46 & 1.30 & 2.43 \\
\hline
\end{tabular}

Table 1. Parameters for Eq. 10 to fit the curves in Fig. 3. The maximum error in the correction factor $f_{\theta, R / \lambda}$ using these parameters is $<3 \%$ for any $R / \lambda$ value in the range 0.1 to 6 .

This new ability to extend the hemispherical cap model to other angles offers the ability to apply it for the first time to measurements which could not be made with normal detection (as is often the case, and always the case with whole classes of analyzer types). However, it goes well beyond this in its advantages, since it also allows more accurate analyses whenever there are strong contributions from elastic scattering effects, such as strong forward-focusing peaks in 
XPS at certain angles due to photoelectron diffraction, which are common for single-crystalline substrates and epitaxial or otherwise ordered adsorbate nanoparticles. Thus, one can now apply this hemispherical cap model to measurements at several angles that include both minima and maxima in the angular distribution, thereby directly testing the sensitivity of the results to these elastic scattering effects, and offering the additional possibility to average results over several angles to minimize their impact on resulting parameters.

If the absolute coverage of the adsorbate is known (for example, when using a well know flux to dose it) and the EAL is known, there is only one parameter in fitting the data to this model if the number density of islands. However, if measurements are made at several coverages, one can also treat the EAL values as parameters, as proposed by Diebold et al. in their

original paper ${ }^{1}$ as a means to overcome complications from elastic scattering in estimating EAL values, at a time before these were available in the NIST Database. ${ }^{12}$

Techniques such as scanning tunneling microscopy (STM) often allow one to measure the number density of islands directly. When this is already known from such measurements, applying this hemispherical cap model to XPS or AES should allow one to determine the absolute coverage of adsorbate (i.e., moles per unit area, if not already known) or to test the deviations of particle shape from that of hemispherical caps of one single size (for example, by taking linear combinations of calculated signals from different sizes).

\section{Acknowledgements}

The authors gratefully acknowledge financial support for this work by the U.S. Department of Energy-Office of Basic Energy Sciences under grant no. DE-FG02-96ER14630.

\section{References}

(1) Diebold, U.; Pan, J. M.; Madey, T. E. Physical Review B 1993, 47, 3868.

(2) Argile, C.; Rhead, G. E. Surf. Sci.Rep. 1989, 10, 277.

(3) Venables, J. A. Surface Science 1994, 299, 798.

(4) Campbell, C. T. Surface Science Reports 1997, 27, 1.

(5) Farmer, J. A.; Campbell, C. T. Science 2010, 329, 933.

(6) Farmer, J. A.; Baricuatro, J. H.; Campbell, C. T. Journal of Physical Chemistry C 2010, 114, 17166. 
(7) Fu, Q.; Wagner, T. Surf. Sci. Rep. 2007, 62, 431.

(8) Tong, W. M.; Williams, R. S. Annu. Rev. Phys. Chem. 1994, 45, 401.

(9) NIST Standard Reference Database 71: NIST Electron Inelastic-Mean-Free-Path Database: Version 1.2, http://www.nist.gov/srd/nist71.cfm

(10) Jablonski, A.; Powell, C. J. Surface Science Reports 2002, 47, 33.

(11) Powell, C. J.; Jablonski, A. Journal of Electron Spectroscopy and Related Phenomena 2010, 178-179, 331.

(12) NIST Standard Reference Database 82: NIST Electron Effective-AttenuationLength Database: Version 1.3, http://www.nist.gov/srd/nist82.cfm

(13) Ertl, G.; Küppers, J. Low Energy Electrons and Surface Chemistry; VCH: Weinheim, Federal Republic of Germany; Deerfield Beach, FL, USA, 1985.

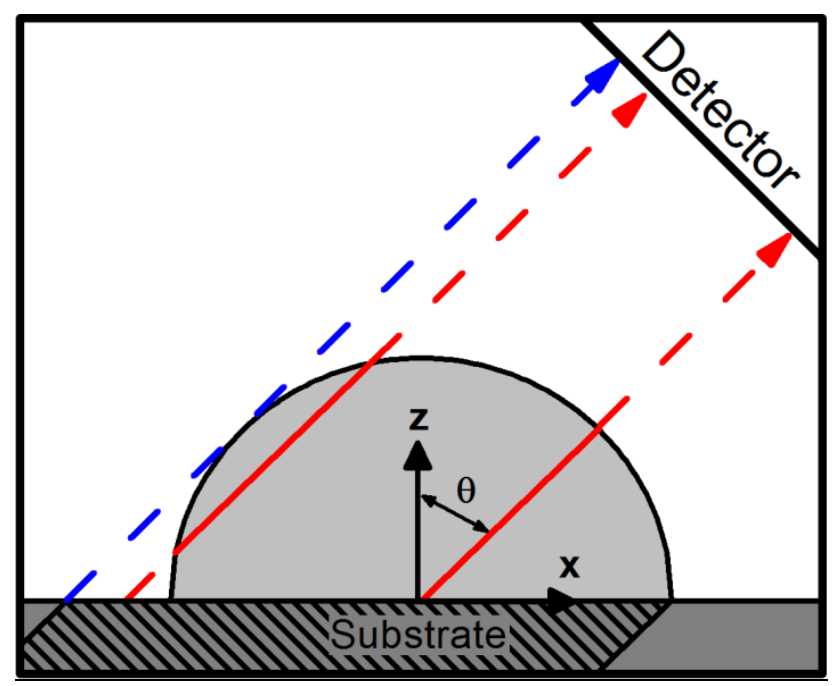

Figure 1. Coordinate system used for derivation of electron intensities from a hemispherical cap of adsorbate and from the underlying substrate when the detector is at the angle shown but infinitely far away compared to the particle size. The y axis (not shown) is out of plane. Electrons emmited from the substrate will be attenuated by the hemispherical cap if they originate from either the area under the cap or from the shadow created by the cap as indicated by the hashed area. The hashed area of the substrate therefore represents the effective spectroscopic footprint of the particle at this particular angle of detection $(\theta$, from normal to the substrate surface). 


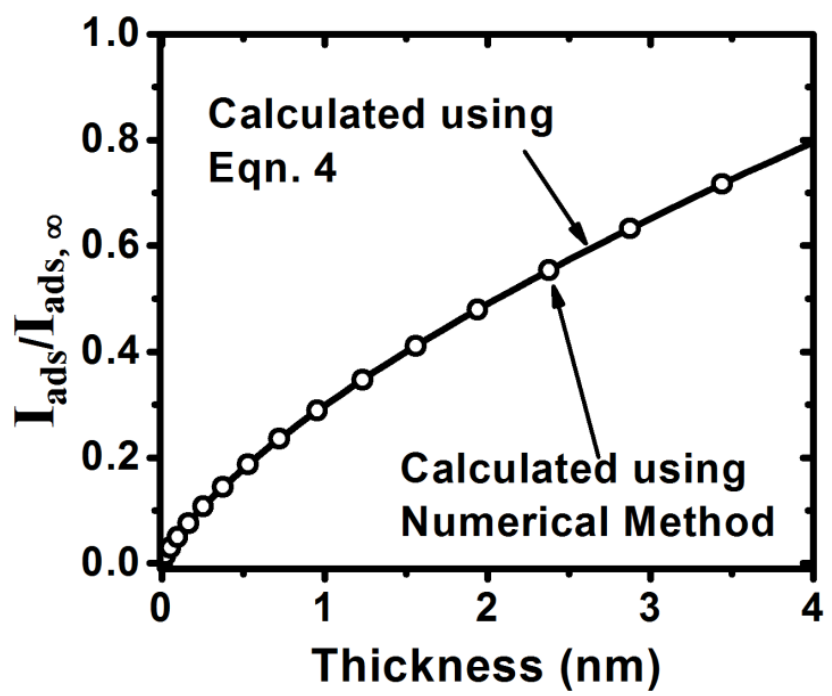

Figure 2. Intensity of adsorbate electrons relative to that for an infinitely thick film versus average thickness for hemispherical caps with number density $n=0.005$ particles $/ \mathrm{nm}^{2}$ and $\lambda=1$ $\mathrm{nm}$. The solid line is calculated using Equation 1 and the open circles were determined using the numerical method with a step size of $0.01 \mathrm{~nm}$. These points each have $<0.1 \%$ deviation from the solid line in their relative intensities. 


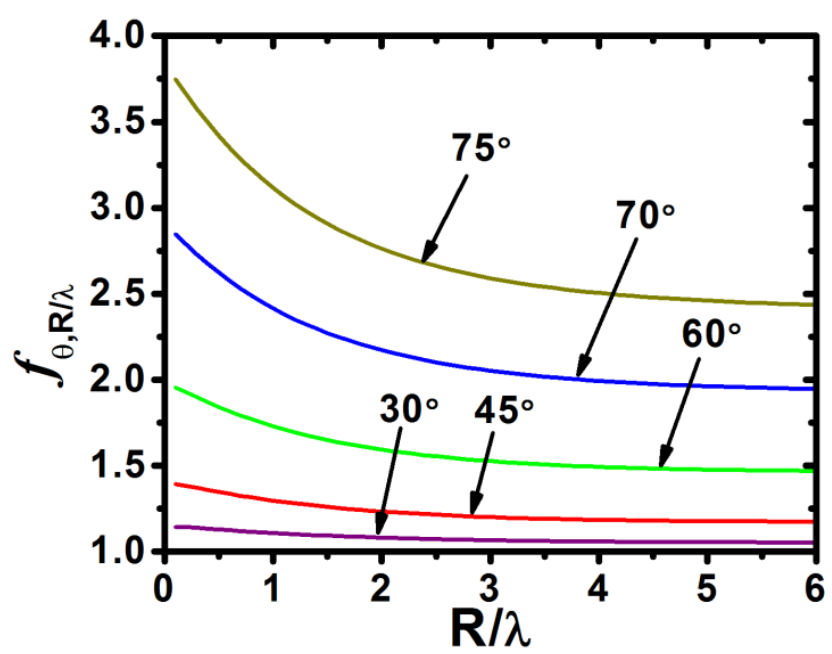

Figure 3. The correction factor $f_{\theta, R / \lambda}=\left(I_{a d s, \theta} / I_{a d s, \theta, \infty}\right) /\left(I_{a d s, \text { normal }} / I_{a d s, \text { normal }, \infty}\right)$ that must be applied to Eq.(4) to calculate adsorbate signals for hemispherical caps measured at other detection angles $\theta$ (relative to their signal for an infinitely thick and continuous adsorbate film) plotted versus the radius of the particle, $R$. Here, $R$ is normalized by the IMFP or EAL for the electron of interest, $\lambda$. When this correction factor is used to correct relative adsorbate signals calculated using Eq. (4) at $\theta=0$ to apply instead to other detection angles, $\lambda=\lambda_{\text {ads }}$ is used here. This same correction factor plotted here can also be used in Eq. (9) to correct relative substrate signals calculated using Eq. (5) at $\theta=0$ to apply instead to data at other detection angles. In that case, one must use $\lambda=\lambda_{\text {sub }}$ here instead. 